\title{
EXACT SOLUTIONS OF KUPERSHMIDT EQUATION, APPROXIMATE SOLUTIONS FOR TIME-FRACTIONAL KUPERSHMIDT EQUATION: A COMPARISON STUDY
}

\section{MEDJAHED DJILALI ${ }^{1, *}$, ALI HAKEM ${ }^{2}$ AND ABDELKADER BENALI ${ }^{3}$}

${ }^{1}$ Oran Higher School of Economics, Laboratory ACEDP, Djillali Liabes University, 22000

\author{
SIDI-BEL-ABBES, Algeria \\ ${ }^{2}$ Laboratory ACEDP, Djillali Liabes University, 22000 SIDI-BEL-ABBES, Algeria \\ ${ }^{3}$ Faculty of The Exact sciences And computer, Mathematics Department, University of Hassiba Benbouali, \\ Chlef 02000, Algeria
}

${ }^{*}$ Corresponding author: djilalimedjahed@yahoo.fr

\begin{abstract}
In this article, a technique namely Tanh method is applied to obtain some traveling wave solutions for Kupershmidt equation, and by using LADM we obtain an approximate solution to timefractional Kupershmidt equation.

A comparison between the traveling wave solution (exact solution) and the approximate one of equation under study, indicate that Laplace Adomian Decomposition Method (LADM) is highly accurate and can be considered a very useful and valuable method.
\end{abstract}

\section{INTRODUCTION}

The study of nonlinear evolution equations have attracted attention of many mathematicians and physicists. Many authors are interested to the research of the exact solutions [9,21,30], because the exact solutions to nonlinear evolution equations are the key tool to understand the various physical phenomena that govern the real world today. Hence, searching for exact traveling wave solutions to nonlinear evolution

Received February $19^{\text {th }}, 2020$; accepted March $16^{\text {th }}, 2020$; published May $1^{\text {st }}, 2020$.

2010 Mathematics Subject Classification. 83C15, 35C07, 81Q05, 35L05, 47J35.

Key words and phrases. Adomian polynomials; Caputo's fractional derivative; Kupershmidt equation; Laplace transform Adomian decomposition method; Tanh method.

(C)2020 Authors retain the copyrights of their papers, and all open access articles are distributed under the terms of the Creative Commons Attribution License. 
equations plays an important role in the study of nonlinear physical phenomena in many fields such as fluid dynamics, water wave mechanics, meteorology, electromagnetic theory, plasma physics and nonlinear optics $[9,21]$.

In this paper, we will study an important nonlinear evolution equation called kueprshmidt equation $($ see $[12,13])$ in the form

$$
u_{t}-u_{5 x}-\frac{5}{2} u u_{3 x}-\frac{25}{4} u_{x} u_{2 x}-\frac{5}{4} u^{2} u_{x}=0 .
$$

Many researchers have studied the general fifth order KdV equation in different contexts:

$$
u_{t}+\omega u_{5 x}+\alpha u u_{3 x}+\beta u_{x} u_{2 x+}+\gamma u^{2} u_{x}=0
$$

where $\omega, \alpha, \beta$ and $\gamma$ real constants. This class includes the generalized Kaup-Kupershmidt equation [34]

$$
u_{t}+20 a^{2} b u_{5 x}+10 a b u u_{3 x}+25 a b u_{x} u_{2 x}+b u^{2} u_{x}=0 .
$$

As the constants $a \neq 0, b \neq 0$ take different values, we retrieve different types of Kaup-Kupershmidt equation. For examples, in the case $a=\frac{1}{20}, b=30$ see [11,16,39], for $a=\frac{1}{60}, b=180$, see [38], Reyes [32] studied the case $a=\frac{1}{10}, b=-5$, if we take $a=-\frac{1}{30}, b=45$ we will find the equation studied by Parker [19,28], and when $a=\frac{1}{30}, b=5$ we get the equation treated in $[7,17]$. While, we obtain the Kupershmidt equation (the equation under study) by taking $a=\frac{1}{5}, b=-\frac{5}{4}$.

To investigate the traveling wave solutions (soliton solutions) [10,18], we propose in this work the Tanh method (or hyperbolic tangent method), because it is a powerful technique to search for traveling waves coming out from one-dimensional nonlinear wave and evolution equations. In particular, in those problems where dispersive effects, reaction, diffusion and/or convection play an important role. To show the strength of the method, an overview is given to find out which kind of problems are solved with this technique and how in some nontrivial cases this method, adapted to the problem at hand, still can be applied. Single as well as coupled equations, arising from wave phenomena which appears in different scientific domains such as physics, chemical kinetics, geochemistry and mathematical biology [15, 24, 25, 45].

But some evolution problems do not admit the traveling wave solutions, due to that, we propose a semianalytical method called Laplace Adomian Decomposition Method (LADM), it is a combination of the Adomian Decomposition Method (ADM) and Laplace transforms. This method was successfully used for solving different problems in $[5,8,14,18,20,23,37,40]$. The ADM was introduced by Adomian [1-4] and has been applied to a wide class of problems in physics, biology and chemical reactions. The method provides the solution in a rapid convergent series with computable terms. The underlying idea of the technique is to assume an infinite solution of the form $u=\sum_{n=0}^{\infty} u_{n}$, then apply Laplace transformation to the differential equation. The nonlinear terms are then decomposed in terms of Adomian polynomials $[6,41,42]$ and an 
iterative algorithm is constructed for the determination of the $u_{n}$ in a recursive manner.

Our goal is to obtained the approximate solutions of the time-fractional Kupershmidt equation, and compare this solution (in particular case) with the traveling wave solution of the equation to show that the proposed algorithm (LADM) is suitable for such problems and is very efficient.

\section{Preliminaries}

Before the beginning of this research, we are trying in a hurry to get to know the supporting materials to accomplish this work.

2.1. The Tanh method. The non-linear wave and evolution equations (in principle, in one dimension) are commonly written as:

$$
u_{t}=\left[u, u_{x}, u_{x x}, \ldots\right] \text { or } u_{t t}=\left[u, u_{x}, u_{x x}, \ldots\right]
$$

We like to know whether traveling waves (or stationary waves) are solutions of (2.1). The first step is to unite the independent variables $\mathrm{x}$ and $\mathrm{t}$ into one particular variable through the definition $\zeta=c(x-\mu t)$. Here $c(>0)$ represents the wave number and $\mu$ is the (unknown) velocity of the traveling wave. Accordingly, the quantity $u(x ; t)$ is replaced by $U(\zeta)$, so that we deal with ODEs, rather than with PDEs. In this way, equations like (2.1) are transformed into

$$
-c \mu \frac{d U}{d \zeta}=\left[U, c \frac{d U}{d \zeta}, \frac{d^{2} U}{d \zeta^{2}}, \ldots\right] \text { or } c^{2} \mu^{2} \frac{d^{2} U}{d \zeta^{2}}=\left[U, c \frac{d U}{d \zeta}, \frac{d^{2} U}{d \zeta^{2}}, \ldots\right]
$$

Our main goal is to derive exact or at least approximate solutions, if possible, for these ODEs. So we introduce a new variable $\Phi=\tanh \zeta$ in the ODE. The latter equation then solely depends on $\Phi$, because all derivatives $\frac{d}{d \zeta}$ in $(2.2)$ are now replaced by $\left(1-\Phi^{2}\right) \frac{d}{d \zeta}$ The solution(s) we are looking for, will be written as a finite power series in $\Phi$

$$
F(\Phi)=\sum_{n=0}^{N} a_{n} \Phi^{n}
$$

To determine $N$ (highest order of $\Phi$ ), the following balancing procedure is used.At least two terms proportional to $\Phi^{N}$ must appear after substitution of ansatz (2.3) into the equation under study. As a result of this analysis, we definitely require $a_{N+1}=0$ and $a_{N} \neq 0$ for a particular $\mathrm{N}$. It turns out that $N=1$ or 2 in most cases. This balance (and thus $N$ ) is obtained by comparing the behavior of $\Phi^{N}$ in the highest derivative against its counterpart within the nonlinear term(s). As soon as $N$ is determined in this way, we get after substitution of (2.3) into(2.2) (transformed to the $\Phi$ variable) algebraic equations for $a_{n}(n=0 ; 1 ; \ldots ; N)$. Depending on the problem under study, the wave number $c$ will remain fixed or undetermined. As already mentioned, the velocity $\mu$ of the traveling wave is always a function of $c$. If one is able to find nontrivial values for $a_{n}(n=0 ; 1 ; \ldots ; N)$, in terms of known quantities, a solution is ultimately obtained (see [24]). 
2.2. Laplace transform. Given a suitable function $F(t)$ the Laplace transform $[35,36]$, written $f(s)$ is defined by

$$
\mathcal{L}[F(t)]=f(s)=\int_{0}^{\infty} F(t) e^{-s t} d t
$$

the inverse Laplace transform is defined by

$$
\mathcal{L}^{-1}[f(s)]=F(t)
$$

The important properties of Laplace transform and it's inverse that will be used in this paper are :

- If $F_{1}(t)$ and $F_{2}(t)$ are two functions whose Laplace transform exists, then

- $\mathcal{L}\left[a F_{1}(t)+b F_{2}(t)\right]=a \mathcal{L}\left[F_{1}(t)\right]+b \mathcal{L}\left[F_{2}(t)\right]$,

- $\mathcal{L}\left(t^{\alpha}\right)=\Gamma(\alpha+1) s^{-\alpha-1}, \quad \alpha>0$,

- $\mathcal{L}\left(t^{n}\right)=\frac{n !}{s^{n+1}}, \quad n$ a positive integer.

- The inverse Laplace transform is linear, i.e $\mathcal{L}^{-1}\left[a f_{1}(s)+b f_{2}(s)\right]=a \mathcal{L}^{-1}\left[f_{1}(s)\right]+b \mathcal{L}^{-1}\left[f_{2}(s)\right]$,

- $\mathcal{L}^{-1}\left(\frac{1}{s^{\alpha}}\right)=\frac{t^{\alpha-1}}{\Gamma(\alpha)}, \quad \alpha>0$

2.3. Caputo derivative. There exists a vast literature on different definitions of fractional derivatives. The most popular ones are the Riemann-Liouville and the Caputo derivatives. The Caputo derivative of order $\alpha$ is defined by the formula $[22,27,29]$ :

$$
D_{*}^{\alpha} f(t)=\left\{\begin{array}{l}
\frac{1}{\Gamma(m-\alpha)} \int_{0}^{t}(t-\tau)^{m-\alpha-1} f^{(m)}(\tau) d \tau, \quad \text { if } m-1<\alpha<m \\
\frac{d^{m}}{d t^{m}} f(t), \quad \text { if } \alpha=m,
\end{array}\right.
$$

where $m \in \mathbb{N}^{*}$ and $\Gamma($.$) denotes the Gamma function defined by \Gamma(x)=\int_{0}^{\infty} t^{x-1} e^{-t} d t, \quad x>0$.

The important properties of the Caputo derivative that will be used in this paper are $[23,26,31,33,43,44]$ :

$$
\begin{gathered}
D^{\alpha} t^{\beta}=\frac{\Gamma(1+\beta)}{\Gamma(1+\beta-\alpha)} t^{\beta-\alpha} \\
D^{\alpha} c=0
\end{gathered}
$$

The Laplace transform of the Caputo derivative is:

$$
\mathcal{L}\left[D_{t}^{\alpha} u(x, t)\right]=s^{\alpha} u(x, s)-\sum_{i=0}^{n-1} u^{(i)}\left(x, 0^{+}\right) s^{\alpha-1-i}, \quad n-1<\alpha \leq n
$$

2.4. The Adomian Decomposition Method Combined with Laplace Transform. The ADM is a method to solve ordinary and nonlinear differential equations. Using this method is possible to express analytic solutions in terms of a series. In a nutshell, the method identifies and separates the linear and nonlinear parts of a differential equation. Inverting and applying the highest order differential operator that is contained in the linear part of the equation, it is possible to express the solution in terms of the rest of the equation affected by the inverse operator. At this point, the solution is proposed by means of a series 
with terms that will be determined and that give rise to the Adomian polynomials. The nonlinear part can also be expressed in terms of these polynomials. The initial (or the border conditions) and the terms that contain the independent variables will be considered as the initial approximation. In this way and by means of a recurrence relations, it is possible to find the terms of the series that give the approximate solution of the differential equation (see [14]). Given a partial (or ordinary) differential equation

$$
F u(x, t)=h(x, t) \text { with initial condition } u(x, 0)=f(x),
$$

where $F$ is a differential operator that could, in general, be nonlinear and therefore includes some linear and nonlinear terms. In general, Eq. (2.10) could be written as

$$
L_{t} u(x, t)=R u(x, t)+N u(x, t)+h(x, t)
$$

where $L_{t}=\frac{\partial^{\alpha}}{\partial t^{\alpha}}, 0<\alpha \leq 1$ (in this paper), $R$ is a linear operator that includes partial derivatives with respect to $x, N$ is a nonlinear operator and $h$ is a non-homogeneous term that is $u$-independent. The LADM consists of applying Laplace transform first on both sides of Eq. (2.11), obtaining

$$
\mathcal{L}\left\{L_{t} u(x, t)\right\}=\mathcal{L}\{R u(x, t)+N u(x, t)+h(x, t)\}
$$

An equivalent expression to $(5)$ is

$$
s^{\alpha} u(x, s)-u(x, 0) s^{\alpha-1}=\mathcal{L}\{R u(x, t)+N u(x, t)+h(x, t)\} .
$$

In the homogeneous case, $h(x, t)=0$, we have

$$
u(x, s)=\frac{f(x)}{s}+\frac{1}{s^{\alpha}} \mathcal{L}\{R u(x, t)+N u(x, t)\} .
$$

now, applying the inverse Laplace transform to E q. (2.14)

$$
u(x, t)=f(x)+\mathcal{L}^{-1}\left[\frac{1}{s^{\alpha}} \mathcal{L}\{R u(x, t)+N u(x, t)\}\right] .
$$

The ADM method proposes a series solution $\mathrm{u}(\mathrm{x}, \mathrm{t})$ given by,

$$
u(x, t)=\sum_{n=0}^{\infty} u_{n}(x, t) .
$$

The nonlinear term $N u(x, t)$ is given by

$$
N u(x, t)=\sum_{n=0}^{\infty} P_{n}\left(u_{0}, u_{1}, u_{2}, \ldots, u_{n}\right) .
$$

where $\left\{A_{n}\right\}_{n=0}^{\infty}$ is the so-called Adomian polynomials sequence established in [42], in general, give us term to term: 


$$
\begin{aligned}
& P_{0}=N\left(u_{0}\right) \\
& P_{1}=u_{1} N^{\prime}\left(u_{0}\right) \\
& P_{2}=u_{2} N^{\prime}\left(u_{0}\right)+\frac{1}{2} u_{1}^{2} N^{\prime \prime}\left(u_{0}\right) \\
& P_{3}=u_{3} N^{\prime}\left(u_{0}\right)+u_{1} u_{2} N^{\prime \prime}\left(u_{0}\right)+\frac{1}{3 !} u_{1}^{3} N^{(3)}\left(u_{0}\right) \\
& P_{4}=u_{4} N^{\prime}\left(u_{0}\right)+\left(\frac{1}{2} u_{2}^{2}+u_{1} u_{3}\right) N^{\prime \prime}\left(u_{0}\right)+\frac{1}{2 !} u_{1}^{2} u_{2} N^{(3)}\left(u_{0}\right)+\frac{1}{4 !} u_{1}^{4} N^{(4)}\left(u_{0}\right)
\end{aligned}
$$

Other polynomials can be generated in a similar way. Some other approaches to obtain Adomian's polynomials can be found in [42].

Using (2.16) and (2.17) into E q. (2.15), we obtain,

$$
\sum_{n=0}^{\infty} u_{n}(x, t)=f(x)+\mathcal{L}^{-1}\left[\frac{1}{s^{\alpha}} \mathcal{L}\left\{R \sum_{n=0}^{\infty} u_{n}(x, t)+\sum_{n=0}^{\infty} P_{n}\left(u_{0}, u_{1}, u_{2}, \ldots, u_{n}\right)\right\}\right] .
$$

we deduce the following recurrence formulas

$$
\left\{\begin{array}{l}
u_{0}(x, t)=f(x) \\
u_{n+1}(x, t)=\mathcal{L}^{-1}\left[\frac{1}{s^{\alpha}} \mathcal{L}\left\{R u_{n}(x, t)+P_{n}\left(u_{0}, u_{1}, u_{2}, \ldots, u_{n}\right)\right\}\right], n=0,1,2 \ldots
\end{array}\right.
$$

Using (2.19) we can obtain an approximate solution of (2.10), using

$$
u(x, t) \approx \sum_{n=0}^{k} u_{n}(x, t), \text { where } \lim _{t \rightarrow \infty} \sum_{n=0}^{k} u_{n}(x, t)=u(x, t)
$$

Remark 2.1. All results and plots bellow are obtained by using Mathematica software.

\section{MAin Results}

3.1. Kupershmidt equation solutions by using Tanh method. in this section, we will apply the Tanh method to find the axact solutions of Kupershmidt equation in the form,

$$
\frac{\partial u(x, t)}{\partial t}-\frac{\partial^{5} u(x, t)}{\partial x^{5}}-\frac{5}{2} u(x, t) \frac{\partial^{3} u(x, t)}{\partial x^{3}}-\frac{25}{4} \frac{\partial u(x, t)}{\partial x} \frac{\partial^{2} u(x, t)}{\partial x^{2}}-\frac{5}{4} u(x, t)^{2} \frac{\partial u(x, t)}{\partial x}=0
$$

We consider the traveling wave transformation defined by,

$$
U(\zeta)=u(x, t), \zeta=c(x-\mu t)
$$

Using traveling wave Eqs. (3.2), then (3.1) transform into the following ordinary differential equations

$$
\mu \mathrm{U}^{(1)}+c^{4} U^{(5)}+\frac{5}{2} c^{2} U^{(3)} U+\frac{25}{4} c^{2} U^{(2)} U^{(1)}+\frac{5}{4} U^{2} U^{(1)}=0
$$


Now balancing the highest order derivative $U^{(5)}$ and nonlinear term $U^{(2)} U^{(1)}$, we get $2 N+3=N+5$ or equivalent to $N=2$. Therefore, Eq. (2.3) reduces to

$$
U(\zeta)=a_{0}+a_{1} \tanh (\zeta)+a_{2} \tanh ^{2}(\zeta)
$$

substituting Eq. (3.4) into Eq. (3.3) and using Mathematica software we get a polynomial of tanh $(\zeta)^{k}$, $(\mathrm{k}$ $=0,1,2, \ldots)$. Equating the coefficients of this polynomial of the same powers of tanh $(\zeta)$ to zero, we obtain a system of algebraic equations for $a_{0}, a_{1}, a_{2}, \mu$ and $c$.

$$
\begin{aligned}
& -16 a_{1} c^{5}+5 a_{0} a_{1} c^{3}-\frac{25}{2} a_{1} a_{2} c^{3}-a_{1} c \mu-\frac{5}{4} a_{0}^{2} a_{1} c=0 \\
& -272 a_{2} c^{5}+\frac{35}{2} a_{1}^{2} c^{3}-25 a_{2}^{2} c^{3}+40 a_{0} a_{2} c^{3}-2 a_{2} c \mu-\frac{5}{2} a_{0} a_{1}^{2} c-\frac{5}{2} a_{0}^{2} a_{2} c=0 \\
& 136 a_{1} c^{5}-20 a_{0} a_{1} c^{3}+\frac{265}{2} a_{1} a_{2} c^{3}+a_{1} c \mu-\frac{5 a_{1}^{3} c}{4}+\frac{5}{4} a_{0}^{2} a_{1} c-\frac{15}{2} a_{0} a_{1} a_{2} c=0 \\
& 1232 a_{2} c^{5}-45 a_{1}^{2} c^{3}+165 a_{2}^{2} c^{3}-100 a_{0} a_{2} c^{3}+2 a_{2} c \mu+\frac{5}{2} a_{0} a_{1}^{2} c-5 a_{0} a_{2}^{2} c+\frac{5}{2} a_{0}^{2} a_{2} c-5 a_{1}^{2} a_{2} c=0 \\
& -240 a_{1} c^{5}+15 a_{0} a_{1} c^{3}-\frac{515}{2} a_{1} a_{2} c^{3}+\frac{5 a_{1}^{3} c}{4}-\frac{25}{4} a_{1} a_{2}^{2} c+\frac{15}{2} a_{0} a_{1} a_{2} c=0 \\
& -1680 a_{2} c^{5}++\frac{55}{2} a_{1}^{2} c^{3}-275 a_{2}^{2} c^{3}+60 a_{0} a_{2} c^{3}-\frac{5 a_{2}^{3} c}{2}+5 a_{0} a_{2}^{2} c+5 a_{1}^{2} a_{2} c=0 \\
& 120 a_{1} c^{5}+\frac{275}{2} a_{1} a_{2} c^{3}+\frac{25}{4} a_{1} a_{2}^{2} c=0 \\
& 720 a_{2} c^{5}+135 a_{2}^{2} c^{3}+\frac{5 a_{2}^{3} c}{2}=0
\end{aligned}
$$

where $a_{2} \neq 0$.

Solving them by means of Mathematica gives:

$$
\begin{aligned}
& \left\{a_{0} \rightarrow 4 c^{2}, a_{1} \rightarrow 0, a_{2} \rightarrow-6 c^{2}, \mu \rightarrow-c^{4}\right\}, \\
& \left\{a_{0} \rightarrow 32 c^{2}, a_{1} \rightarrow 0, a_{2} \rightarrow-48 c^{2}, \mu \rightarrow-176 c^{4}\right\},
\end{aligned}
$$

substituting into Eq. (3.4), it follows

$$
\begin{gathered}
u_{1}(x, t)=4 c^{2}-6 c^{2} \tanh ^{2}\left(c x+c^{5} t\right), \\
u_{2}(x, t)=32 c^{2}-48 c^{2} \tanh ^{2}\left(c x+176 c^{5} t\right),
\end{gathered}
$$

3.2. The approximate solution of time-fractional Kupershmidt equation by LADM. Consider the time-fractional Kupershmidt equation

$$
\frac{\partial^{\alpha}}{\partial t^{\alpha}} u(x, t)=\frac{\partial^{5} u(x, t)}{\partial x^{5}}+\frac{5}{2} u(x, t) \frac{\partial^{3} u(x, t)}{\partial x^{3}}+\frac{25}{4} \frac{\partial u(x, t)}{\partial x} \frac{\partial^{2} u(x, t)}{\partial x^{2}}+\frac{5}{4} u(x, t)^{2} \frac{\partial u(x, t)}{\partial x}
$$

subject to the initial conditions

$$
u(x, 0)=f(x)=4 c^{2}-6 c^{2} \tanh ^{2}(c x)
$$


where $0<\alpha \leq 1$ and $\frac{\partial^{\alpha}}{\partial t^{\alpha}}=D_{t}^{\alpha}$ the derivatives in the sens of Caputo.

Comparing (3.7) with Eq. (2.11) we have that $h(x, t)=0, L_{t}$ and $R$ becomes:

$$
L_{t} u=D_{t}^{\alpha} u=\frac{\partial^{\alpha}}{\partial t^{\alpha}} u, \quad R(u)=\frac{\partial^{5} u(x, t)}{\partial x^{5}}=u_{5 x}(x, t),
$$

while the nonlinear term are given by

$$
\begin{aligned}
N u & =\frac{5}{2} u(x, t) \frac{\partial^{3} u(x, t)}{\partial x^{3}}+\frac{25}{4} \frac{\partial u(x, t)}{\partial x} \frac{\partial^{2} u(x, t)}{\partial x^{2}}+\frac{5}{4} u(x, t)^{2} \frac{\partial u(x, t)}{\partial x} \\
& :=\frac{5}{2} u(x, t) u_{3 x}(x, t)+\frac{25}{4} u_{x}(x, t) u_{2 x}(x, t)+\frac{5}{4} u(x, t)^{2} u_{x}(x, t),
\end{aligned}
$$

By using now Eq. (2.19) through the LADM method we obtain recursively

$$
\left\{\begin{array}{l}
u_{0}(x, t)=f(x) \\
u_{n+1}(x, t)=\mathcal{L}^{-1}\left[\frac{1}{s^{\alpha}} \mathcal{L}\left\{R\left(u_{n}\right)+P_{n}\left(u_{0}, u_{1}, u_{2}, \ldots, u_{n}\right)\right\}\right], n=0,1,2 \ldots
\end{array}\right.
$$

from this, we will consider the decomposition of the nonlinear terms into Adomian polynomials as

$$
N u=N_{1} u+N_{2} u+N_{3} u=\sum_{n=0}^{\infty} P_{n}\left(u_{0}, u_{1}, u_{2}, \ldots, u_{n}\right) .
$$

Let

$$
\begin{gathered}
N_{1} u=\frac{5}{2} u(x, t) u_{3 x}(x, t)=\frac{5}{2} \sum_{n=0}^{\infty} u_{n} \sum_{n=0}^{\infty} u_{n_{3 x}}=\sum_{n=0}^{\infty} A_{n}\left(u_{0}, u_{1}, u_{2}, \ldots, u_{n}\right), \\
N_{2} u=\frac{25}{4} u_{x}(x, t) u_{2 x}(x, t)=\frac{25}{4} \sum_{n=0}^{\infty} u_{n_{x}} \sum_{n=0}^{\infty} u_{n_{2 x}}=\sum_{n=0}^{\infty} B_{n}\left(u_{0}, u_{1}, u_{2}, \ldots, u_{n}\right), \\
N_{3} u=\frac{5}{4} u(x, t)^{2} u_{x}(x, t)=\frac{5}{4}\left(\sum_{n=0}^{\infty} u_{n}\right)^{2} * \sum_{n=0}^{\infty} u_{n_{x}}=\sum_{n=0}^{\infty} C_{n}\left(u_{0}, u_{1}, u_{2}, \ldots, u_{n}\right),
\end{gathered}
$$

where $P_{n}=A_{n}+B_{n}+C_{n}$.

Using ADM, Eq.(2.16) gives

$$
u(x, t)=\sum_{n=0}^{\infty} u_{n}(x, t)
$$

thus, the Adomian polynomials $A_{n}$ are in the forms

$$
\begin{aligned}
A_{0} & =\frac{5}{2} u_{0} u_{0_{3 x}} \\
A_{1} & =\frac{5}{2} u_{1} u_{0_{3 x}}+\frac{5}{2} u_{0} u_{1_{3 x}} \\
A_{2} & =\frac{5}{2} u_{2} u_{0_{3 x}}+\frac{5}{2} u_{1} u_{1_{3 x}}+\frac{5}{2} u_{0} u_{2_{3 x}} \\
A_{3} & =\frac{5}{2} u_{3} u_{0_{3 x}}+\frac{5}{2} u_{2} u_{1_{3 x}}+\frac{5}{2} u_{1} u_{2_{3 x}}+\frac{5}{2} u_{0} u_{3_{3 x}} \\
A_{4} & =\frac{5}{2} u_{4} u_{0_{3 x}}+\frac{5}{2} u_{3} u_{1_{3 x}}+\frac{5}{2} u_{2} u_{2_{3 x}}+\frac{5}{2} u_{1} u_{3_{3 x}}+\frac{5}{2} u_{0} u_{4_{3 x}}
\end{aligned}
$$




$$
\begin{aligned}
& B_{0}=\frac{25}{4} u_{0_{x}} u_{0_{2 x}} \\
& B_{1}=\frac{25}{4} u_{1_{x}} u_{0_{2 x}}+\frac{25}{4} u_{0_{x}} u_{1_{2 x}} \\
& B_{2}=\frac{25}{4} u_{2_{x}} u_{0_{2 x}}+\frac{25}{4} u_{1_{x}} u_{1_{2 x}}+\frac{25}{4} u_{0_{x}} u_{2_{2 x}} \\
& B_{3}=\frac{25}{4} u_{3_{x}} u_{0_{2 x}}+\frac{25}{4} u_{2_{x}} u_{1_{2 x}}+\frac{25}{4} u_{1_{x}} u_{2_{2 x}}+\frac{25}{4} u_{0_{x}} u_{3_{2 x}} \\
& B_{4}=\frac{25}{4} u_{4_{x}} u_{0_{2 x}}+\frac{25}{4} u_{3_{x}} u_{1_{2 x}}+\frac{25}{4} u_{2_{x}} u_{2_{2 x}}+\frac{25}{4} u_{1_{x}} u_{3_{2 x}}+\frac{25}{4} u_{0_{x}} u_{4_{2 x}},
\end{aligned}
$$

and

$$
\begin{aligned}
C_{0} & =\frac{5}{4} u_{0}^{2} u_{0_{x}}, \\
C_{1} & =\frac{5}{4} u_{0}^{2} u_{1_{x}}+\frac{5}{2} u_{1} u_{0} u_{0_{x}} \\
C_{2} & =\frac{5}{4} u_{0}^{2} u_{2_{x}}+\frac{5}{2} u_{2} u_{0} u_{0_{x}}+\frac{5}{4} u_{1}^{2} u_{0_{x}} \\
C_{3} & =\frac{5}{4} u_{0}^{2} u_{3_{x}}+\frac{5}{2} u_{3} u_{0} u_{0_{x}}+\frac{5}{2} u_{2} u_{0} u_{1_{x}}+\frac{5}{2} u_{1} u_{0} u_{2_{x}}+\frac{5}{2} u_{1} u_{2} u_{0_{x}}+\frac{5}{4} u_{1}^{2} u_{1_{x}} \\
C_{4} & =\frac{5}{4} u_{0}^{2} u_{4_{x}}+\frac{5}{2} u_{4} u_{0} u_{0_{x}}+\frac{5}{2} u_{2} u_{0} u_{2_{x}}+\frac{5}{4} u_{2}^{2} u_{0_{x}}+\frac{5}{2} u_{1} u_{3} u_{0_{x}}+\frac{5}{2} u_{1} u_{2} u_{1_{x}}+\frac{5}{4} u_{1}^{2} u_{2_{x}} .
\end{aligned}
$$

Through the LADM we obtain recursively

$$
\begin{aligned}
u_{0}(x, t)= & f(x) \\
u_{1}(x, t)= & \mathcal{L}^{-1}\left[\frac{1}{s^{\alpha}} \mathcal{L}\left\{u_{0_{5 x}}+A_{0}+B_{0}+C_{0}\right\}\right] \\
u_{2}(x, t)= & \mathcal{L}^{-1}\left[\frac{1}{s^{\alpha}} \mathcal{L}\left\{u_{1_{5 x}}+A_{1}+B_{1}+C_{1}\right\}\right] \\
u_{3}(x, t)= & \mathcal{L}^{-1}\left[\frac{1}{s^{\alpha}} \mathcal{L}\left\{u_{2_{5 x}}+A_{2}+B_{2}+C_{2}\right\}\right] \\
\vdots & \vdots \\
u_{n+1}(x, t)= & \mathcal{L}^{-1}\left[\frac{1}{s^{\alpha}} \mathcal{L}\left\{u_{n_{5 x}}+A_{n}+B_{n}+C_{n}\right\}\right]
\end{aligned}
$$


Besides

$$
\begin{aligned}
A_{0}= & -1440 c^{7} \tanh ^{3}(c x) \operatorname{sech}^{4}(c x)+960 c^{7} \tanh (c x) \operatorname{sech}^{4}(c x)+720 c^{7} \tanh ^{5}(c x) \operatorname{sech}^{2}(c x) \\
& -480 c^{7} \tanh ^{3}(c x) \operatorname{sech}^{2}(c x) \\
B_{0}= & 900 c^{7} \tanh (c x) \operatorname{sech}^{6}(c x)-1800 c^{7} \tanh ^{3}(c x) \operatorname{sech}^{4}(c x) \\
C_{0}= & -540 c^{7} \tanh ^{5}(c x) \operatorname{sech}^{2}(c x)+720 c^{7} \tanh ^{3}(c x) \operatorname{sech}^{2}(c x)-240 c^{7} \tanh (c x) \operatorname{sech}^{2}(c x) .
\end{aligned}
$$

With the above, we have

$$
\begin{aligned}
u_{0}(x, t)= & 4 c^{2}-6 c^{2} \tanh ^{2}(c x) \\
u_{1}(x, t)= & -\frac{732 c^{7} t^{\alpha} \tanh (c x) \operatorname{sech}^{6}(c x)}{\Gamma(\alpha+1)}-\frac{744 c^{7} t^{\alpha} \tanh ^{3}(c x) \operatorname{sech}^{4}(c x)}{\Gamma(\alpha+1)}+\frac{960 c^{7} t^{\alpha} \tanh (c x) \operatorname{sech}^{4}(c x)}{\Gamma(\alpha+1)} \\
& -\frac{12 c^{7} t^{\alpha} \tanh ^{5}(c x) \operatorname{sech}^{2}(c x)}{\Gamma(\alpha+1)}+\frac{240 c^{7} t^{\alpha} \tanh ^{3}(c x) \operatorname{sech}^{2}(c x)}{\Gamma(\alpha+1)}-\frac{240 c^{7} t^{\alpha} \tanh (c x) \operatorname{sech}^{2}(c x)}{\Gamma(\alpha+1)}
\end{aligned}
$$

and proceeding in a similar way we get

$$
\begin{aligned}
& A_{1}=\frac{101760 c^{12} t^{\alpha} \operatorname{sech}^{10}(c x)}{\Gamma(\alpha+1)}-\frac{328320 c^{12} t^{\alpha} \tanh ^{2}(c x) \operatorname{sech}^{10}(c x)}{\Gamma(\alpha+1)}+\frac{1188720 c^{12} t^{\alpha} \tanh ^{4}(c x) \operatorname{sech}^{8}(c x)}{\Gamma(\alpha+1)} \\
& -\frac{120000 c^{12} t^{\alpha} \operatorname{sech}^{8}(c x)}{\Gamma(\alpha+1)}-\frac{442560 c^{12} t^{\alpha} \tanh ^{2}(c x) \operatorname{sech}^{8}(c x)}{\Gamma(\alpha+1)}+\frac{833760 c^{12} t^{\alpha} \tanh ^{6}(c x) \operatorname{sech}^{6}(c x)}{\Gamma(\alpha+1)} \\
& \frac{19200 c^{12} t^{\alpha} \operatorname{sech}^{6}(c x)}{\Gamma(\alpha+1)}-\frac{2111040 c^{12} t^{\alpha} \tanh ^{4}(c x) \operatorname{sech}^{6}(c x)}{\Gamma(\alpha+1)}+\frac{950400 c^{12} t^{\alpha} \tanh ^{2}(c x) \operatorname{sech}^{6}(c x)}{\Gamma(\alpha+1)} \\
& -\frac{684720 c^{12} t^{\alpha} \tanh ^{8}(c x) \operatorname{sech}^{4}(c x)}{\Gamma(\alpha+1)}+\frac{990240 c^{12} t^{\alpha} \tanh ^{6}(c x) \operatorname{sech}^{4}(c x)}{\Gamma(\alpha+1)} \\
& -\frac{187200 c^{12} t^{\alpha} \tanh ^{4}(c x) \operatorname{sech}^{4}(c x)}{\Gamma(\alpha+1)}-\frac{105600 c^{12} t^{\alpha} \tanh ^{2}(c x) \operatorname{sech}^{4}(c x)}{\Gamma(\alpha+1)}-\frac{1440 c^{12} t^{\alpha} \tanh ^{10}(c x) \operatorname{sech}^{2}(c x)}{\Gamma(\alpha+1)} \\
& +\frac{29760 c^{12} t^{\alpha} \tanh ^{8}(c x) \operatorname{sech}^{2}(c x)}{\Gamma(\alpha+1)}-\frac{48000 c^{12} t^{\alpha} \tanh ^{6}(c x) \operatorname{sech}^{2}(c x)}{\Gamma(\alpha+1)}+\frac{19200 c^{12} t^{\alpha} \tanh ^{4}(c x) \operatorname{sech}^{2}(c x)}{\Gamma(\alpha+1)} \\
& B_{1}=\frac{54900 c^{12} t^{\alpha} \operatorname{sech}^{12}(c x)}{\Gamma(\alpha+1)}-\frac{72000 c^{12} t^{\alpha} \operatorname{sech}^{10}(c x)}{\Gamma(\alpha+1)}+\frac{18000 c^{12} t^{\alpha} \operatorname{sech}^{8}(c x)}{\Gamma(\alpha+1)} \\
& -\frac{1035000 c^{12} t^{\alpha} \tanh ^{2}(c x) \operatorname{sech}^{10}(c x)}{\Gamma(\alpha+1)}+\frac{202500 c^{12} t^{\alpha} \tanh ^{4}(c x) \operatorname{sech}^{8}(c x)}{\Gamma(\alpha+1)} \\
& +\frac{1278000 c^{12} t^{\alpha} \tanh ^{2}(c x) \operatorname{sech}^{8}(c x)}{\Gamma(\alpha+1)}+\frac{1299600 c^{12} t^{\alpha} \tanh ^{6}(c x) \operatorname{sech}^{6}(c x)}{\Gamma(\alpha+1)} \\
& -\frac{1224000 c^{12} t^{\alpha} \tanh ^{4}(c x) \operatorname{sech}^{6}(c x)}{\Gamma(\alpha+1)}-\frac{216000 c^{12} t^{\alpha} \tanh ^{2}(c x) \operatorname{sech}^{6}(c x)}{\Gamma(\alpha+1)} \\
& +\frac{7200 c^{12} t^{\alpha} \tanh ^{8}(c x) \operatorname{sech}^{4}(c x)}{\Gamma(\alpha+1)}-\frac{144000 c^{12} t^{\alpha} \tanh ^{6}(c x) \operatorname{sech}^{4}(c x)}{\Gamma(\alpha+1)} \\
& +\frac{144000 c^{12} t^{\alpha} \tanh ^{4}(c x) \operatorname{sech}^{4}(c x)}{\Gamma(\alpha+1)}
\end{aligned}
$$




$$
\begin{aligned}
C_{1}= & -\frac{14640 c^{12} t^{\alpha} \operatorname{sech}^{8}(c x)}{\Gamma(\alpha+1)}+\frac{19200 c^{12} t^{\alpha} \operatorname{sech}^{6}(c x)}{\Gamma(\alpha+1)}-\frac{4800 c^{12} t^{\alpha} \operatorname{sech}^{4}(c x)}{\Gamma(\alpha+1)} \\
& -\frac{164700 c^{12} t^{\alpha} \tanh ^{4}(c x) \operatorname{sech}^{8}(c x)}{\Gamma(\alpha+1)}+\frac{131760 c^{12} t^{\alpha} \tanh ^{2}(c x) \operatorname{sech}^{8}(c x)}{\Gamma(\alpha+1)} \\
& -\frac{36720 c^{12} t^{\alpha} \tanh ^{6}(c x) \operatorname{sech}^{6}(c x)}{\Gamma(\alpha+1)}+\frac{175680 c^{12} t^{\alpha} \tanh ^{4}(c x) \operatorname{sech}^{6}(c x)}{\Gamma(\alpha+1)} \\
& -\frac{129600 c^{12} t^{\alpha} \tanh ^{2}(c x) \operatorname{sech}^{6}(c x)}{\Gamma(\alpha+1)}+\frac{129060 c^{12} t^{\alpha} \tanh ^{8}(c x) \operatorname{sech}^{4}(c x)}{\Gamma(\alpha+1)} \\
& -\frac{270720 c^{12} t^{\alpha} \tanh ^{6}(c x) \operatorname{sech}^{4}(c x)}{\Gamma(\alpha+1)}+\frac{162720 c^{12} t^{\alpha} \tanh ^{4}(c x) \operatorname{sech}^{4}(c x)}{\Gamma(\alpha+1)} \\
& -\frac{19200 c^{12} t^{\alpha} \tanh ^{2}(c x) \operatorname{sech}^{4}(c x)}{\Gamma(\alpha+1)}+\frac{1080 c^{12} t^{\alpha} \tanh ^{10}(c x) \operatorname{sech}^{2}(c x)}{\Gamma(\alpha+1)} \\
& -\frac{23040 c^{12} t^{\alpha} \tanh ^{8}(c x) \operatorname{sech}^{2}(c x)}{\Gamma(\alpha+1)}+\frac{50880 c^{12} t^{\alpha} \tanh ^{6}(c x) \operatorname{sech}^{2}(c x)}{\Gamma(\alpha+1)} \\
& -\frac{38400 c^{12} t^{\alpha} \tanh ^{4}(c x) \operatorname{sech}^{2}(c x)}{\Gamma(\alpha+1)}+\frac{9600 c^{12} t^{\alpha} \tanh ^{2}(c x) \operatorname{sech}^{2}(c x)}{\Gamma(\alpha+1)},
\end{aligned}
$$

thus,

$$
\begin{aligned}
& u_{2}=\frac{24 c^{12} t^{2 \alpha} \operatorname{sech}^{4}(c x)}{\Gamma(2 \alpha+1)}+\frac{12 c^{12} t^{2 \alpha} \cosh (2 c x) \operatorname{sech}^{4}(c x)}{\Gamma(2 \alpha+1)}, \\
& A_{2}=\frac{115200 c^{17} \operatorname{sech}^{4}(c x) \tanh ^{11}(c x) t^{2 \alpha}}{\Gamma(\alpha+1)^{2}}+\frac{32371200 c^{17} \operatorname{sech}^{6}(c x) \tanh ^{9}(c x) t^{2 \alpha}}{\Gamma(\alpha+1)^{2}} \\
& +\frac{11520000 c^{17} \operatorname{sech}^{10}(c x) \tanh ^{7}(c x) t^{2 \alpha}}{\Gamma(\alpha+1)^{2}}+\frac{149932800 c^{17} \operatorname{sech}^{8}(c x) \tanh ^{7}(c x) t^{2 \alpha}}{\Gamma(\alpha+1)^{2}} \\
& +\frac{2304000 c^{17} \operatorname{sech}^{4}(c x) \tanh ^{7}(c x) t^{2 \alpha}}{\Gamma(\alpha+1)^{2}}+\frac{249523200 c^{17} \operatorname{sech}^{12}(c x) \tanh ^{5}(c x) t^{2 \alpha}}{\Gamma(\alpha+1)^{2}} \\
& +\frac{18316800 c^{17} \operatorname{sech}^{8}(c x) \tanh ^{5}(c x) t^{2 \alpha}}{\Gamma(\alpha+1)^{2}}+\frac{20736000 c^{17} \operatorname{sech}^{6}(c x) \tanh ^{5}(c x) t^{2 \alpha}}{\Gamma(\alpha+1)^{2}} \\
& +\frac{11520 c^{17} \cosh (2 c x) \operatorname{sech}^{4}(c x) \tanh ^{5}(c x) t^{2 \alpha}}{\Gamma(2 \alpha+1)}+\frac{137164320 c^{17} \operatorname{sech}^{14}(c x) \tanh ^{3}(c x) t^{2 \alpha}}{\Gamma(\alpha+1)^{2}} \\
& +\frac{308563200 c^{17} \operatorname{sech}^{10}(c x) \tanh ^{3}(c x) t^{2 \alpha}}{\Gamma(\alpha+1)^{2}}+\frac{6336000 c^{17} \operatorname{sech}^{6}(c x) \tanh ^{3}(c x) t^{2 \alpha}}{\Gamma(\alpha+1)^{2}} \\
& +\frac{23040 c^{17} \operatorname{sech}^{6}(c x) \tanh ^{3}(c x) t^{2 \alpha}}{\Gamma(2 \alpha+1)}+\frac{15360 c^{17} \operatorname{sech}^{4}(c x) \tanh ^{3}(c x) t^{2 \alpha}}{\Gamma(2 \alpha+1)} \\
& +\frac{960 c^{17} \cosh (2 c x) \operatorname{sech}^{4}(c x) \tanh ^{3}(c x) t^{2 \alpha}}{\Gamma(2 \alpha+1)}+\frac{4320 c^{17} \operatorname{sech}^{6}(c x) \sinh (2 c x) \tanh ^{2}(c x) t^{2 \alpha}}{\Gamma(2 \alpha+1)} \\
& +\frac{10080 c^{17} \operatorname{sech}^{4}(c x) \sinh (2 c x) \tanh ^{2}(c x) t^{2 \alpha}}{\Gamma(2 \alpha+1)}+\frac{960 c^{17} \operatorname{sech}^{4}(c x) \sinh (2 c x) t^{2 \alpha}}{\Gamma(2 \alpha+1)} \\
& +\frac{46382400 c^{17} \operatorname{sech}^{14}(c x) \tanh (c x) t^{2 \alpha}}{\Gamma(\alpha+1)^{2}}+\frac{11808000 c^{17} \operatorname{sech}^{10}(c x) \tanh (c x) t^{2 \alpha}}{\Gamma(\alpha+1)^{2}} \\
& +\frac{2880 c^{17} \cosh (2 c x) \operatorname{sech}^{8}(c x) \tanh (c x) t^{2 \alpha}}{\Gamma(2 \alpha+1)}+\frac{6720 c^{17} \cosh (2 c x) \operatorname{sech}^{6}(c x) \tanh (c x) t^{2 \alpha}}{\Gamma(2 \alpha+1)}
\end{aligned}
$$




$$
\begin{aligned}
& -\frac{2880 c^{17} \operatorname{sech}^{4}(c x) \tanh ^{13}(c x) t^{2 \alpha}}{\Gamma(\alpha+1)^{2}}-\frac{1550880 c^{17} \operatorname{sech}^{6}(c x) \tanh ^{11}(c x) t^{2 \alpha}}{\Gamma(\alpha+1)^{2}} \\
& -\frac{83764800 c^{17} \operatorname{sech}^{8}(c x) \tanh ^{9}(c x) t^{2 \alpha}}{\Gamma(\alpha+1)^{2}}-\frac{1267200 c^{17} \operatorname{sech}^{4}(c x) \tanh ^{9}(c x) t^{2 \alpha}}{\Gamma(\alpha+1)^{2}} \\
& -\frac{57772800 c^{17} \operatorname{sech}^{6}(c x) \tanh ^{7}(c x) t^{2 \alpha}}{\Gamma(\alpha+1)^{2}}-\frac{294724800 c^{17} \operatorname{sech}^{10}(c x) \tanh ^{5}(c x) t^{2 \alpha}}{\Gamma(\alpha+1)^{2}} \\
& -\frac{1152000 c^{17} \operatorname{sech}^{4}(c x) \tanh ^{5}(c x) t^{2 \alpha}}{\Gamma(\alpha+1)^{2}}-\frac{366019200 c^{17} \operatorname{sech}^{12}(c x) \tanh ^{3}(c x) t^{2 \alpha}}{\Gamma(\alpha+1)^{2}} \\
& -\frac{86400000 c^{17} \operatorname{sech}^{8}(c x) \tanh ^{3}(c x) t^{2 \alpha}}{\Gamma(\alpha+1)^{2}}-\frac{18622080 c^{17} \operatorname{sech}^{16}(c x) \tanh (c x) t^{2 \alpha}}{\Gamma(\alpha+1)^{2}} \\
& -\frac{38419200 c^{17} \operatorname{sech}^{12}(c x) \tanh (c x) t^{2 \alpha}}{\Gamma(\alpha+1)^{2}}-\frac{1152000 c^{17} \operatorname{sech}^{8}(c x) \tanh (c x) t^{2 \alpha}}{\Gamma(\alpha+1)^{2}} \\
& -\frac{23040 c^{17} \operatorname{sech}^{4}(c x) \tanh ^{5}(c x) t^{2 \alpha}}{\Gamma(2 \alpha+1)}-\frac{17280 c^{17} \operatorname{sech}^{4}(c x) \sinh (2 c x) \tanh ^{4}(c x) t^{2 \alpha}}{\Gamma(2 \alpha+1)} \\
& -\frac{11520 c^{17} \cosh (2 c x) \operatorname{sech}^{6}(c x) \tanh ^{3}(c x) t^{2 \alpha}}{\Gamma(2 \alpha+1)}-\frac{2880 c^{17} \operatorname{sech}^{6}(c x) \sinh (2 c x) t^{2 \alpha}}{\Gamma(2 \alpha+1)} \\
& -\frac{5760 c^{17} \operatorname{sech}^{8}(c x) \tanh (c x) t^{2 \alpha}}{\Gamma(2 \alpha+1)}-\frac{13440 c^{17} \operatorname{sech}^{6}(c x) \tanh (c x) t^{2 \alpha}}{\Gamma(2 \alpha+1)} \\
& -\frac{5760 c^{17} \cosh (2 c x) \operatorname{sech}^{4}(c x) \tanh (c x) t^{2 \alpha}}{\Gamma(2 \alpha+1)} \text {, }
\end{aligned}
$$

$$
\begin{aligned}
B_{2}= & \frac{288000 c^{17} \operatorname{sech}^{4}(c x) \tanh ^{11}(c x) t^{2 \alpha}}{\Gamma(\alpha+1)^{2}}+\frac{54576000 c^{17} \operatorname{sech}^{6}(c x) \tanh ^{9}(c x) t^{2 \alpha}}{\Gamma(\alpha+1)^{2}} \\
& +\frac{431856000 c^{17} \operatorname{sech}^{8}(c x) \tanh ^{7}(c x) t^{2 \alpha}}{\Gamma(\alpha+1)^{2}}+\frac{5760000 c^{17} \operatorname{sech}^{4}(c x) \tanh ^{7}(c x) t^{2 \alpha}}{\Gamma(\alpha+1)^{2}} \\
& +\frac{220665600 c^{17} \operatorname{sech}^{12}(c x) \tanh ^{5}(c x) t^{2 \alpha}}{\Gamma(\alpha+1)^{2}}+\frac{43200000 c^{17} \operatorname{sech}^{6}(c x) \tanh ^{5}(c x) t^{2 \alpha}}{\Gamma(\alpha+1)^{2}} \\
& +\frac{143305200 c^{17} \operatorname{sech}^{14}(c x) \tanh ^{3}(c x) t^{2 \alpha}}{\Gamma(\alpha+1)^{2}}+\frac{360144000 c^{17} \operatorname{sech}^{10}(c x) \tanh ^{3}(c x) t^{2 \alpha}}{\Gamma(\alpha+1)^{2}} \\
& +\frac{7200000 c^{17} \operatorname{sech}^{6}(c x) \tanh ^{3}(c x) t^{2 \alpha}}{\Gamma(\alpha+1)^{2}}+\frac{43200 c^{17} \operatorname{sech}^{6}(c x) \tanh ^{3}(c x) t^{2 \alpha}}{\Gamma(2 \alpha+1)} \\
& +\frac{18000 c^{17} \operatorname{sech}^{6}(c x) \sinh ^{2 \alpha}(2 x) \tanh ^{2}(c x) t^{2 \alpha}}{\Gamma(2 \alpha+1)}+\frac{115956000 c^{17} \operatorname{sech}^{14}(c x) \tanh ^{2 \alpha}(c x) t^{2 \alpha}}{\Gamma(\alpha+1)^{2}} \\
& +\frac{29520000 c^{17} \operatorname{sech}^{10}(c x) \tanh ^{2}(c x) t^{2 \alpha}}{\Gamma(\alpha+1)^{2}}+\frac{7200 c^{17} \cosh ^{2}(2 c x) \operatorname{sech}^{8}(c x) \tanh ^{2}(c x) t^{2 \alpha}}{\Gamma(2 \alpha+1)} \\
& -\frac{7200 c^{17} \operatorname{sech}^{4}(c x) \tanh ^{13}(c x) t^{2 \alpha}}{\Gamma(\alpha+1)^{2}}-\frac{2602800 c^{17} \operatorname{sech}^{6}(c x) \tanh ^{11}(c x) t^{2 \alpha}}{\Gamma(\alpha+1)^{2}} \\
& -\frac{210794400 c^{17} \operatorname{sech}^{8}(c x) \tanh ^{9}(c x) t^{2 \alpha}}{\Gamma(\alpha+1)^{2}}-\frac{3168000 c^{17} \operatorname{sech}^{4}(c x) \tanh ^{9}(c x) t^{2 \alpha}}{\Gamma(\alpha+1)^{2}} \\
& -\frac{177393600 c^{17} \operatorname{sech}^{10}(c x) \tanh ^{7}(c x) t^{2 \alpha}}{\Gamma(\alpha+1)^{2}}-\frac{102096000 c^{17} \operatorname{sech}^{6}(c x) \tanh ^{7}(c x) t^{2 \alpha}}{\Gamma(\alpha+1)^{2}}
\end{aligned}
$$




$$
\begin{aligned}
& -\frac{154908000 c^{17} \operatorname{sech}^{10}(c x) \tanh ^{5}(c x) t^{2 \alpha}}{\Gamma(\alpha+1)^{2}}-\frac{128736000 c^{17} \operatorname{sech}^{8}(c x) \tanh ^{5}(c x) t^{2 \alpha}}{\Gamma(\alpha+1)^{2}} \\
& -\frac{2880000 c^{17} \operatorname{sech}^{4}(c x) \tanh ^{5}(c x) t^{2 \alpha}}{\Gamma(\alpha+1)^{2}}-\frac{416520000 c^{17} \operatorname{sech}^{12}(c x) \tanh ^{3}(c x) t^{2 \alpha}}{\Gamma(\alpha+1)^{2}} \\
& -\frac{95040000 c^{17} \operatorname{sech}^{8}(c x) \tanh ^{3}(c x) t^{2 \alpha}}{\Gamma(\alpha+1)^{2}}-\frac{46555200 c^{17} \operatorname{sech}^{16}(c x) \tanh (c x) t^{2 \alpha}}{\Gamma(\alpha+1)^{2}} \\
& -\frac{96048000 c^{17} \operatorname{sech}^{12}(c x) \tanh (c x) t^{2 \alpha}}{\Gamma(\alpha+1)^{2}}-\frac{2880000 c^{17} \operatorname{sech}^{8}(c x) \tanh (c x) t^{2 \alpha}}{\Gamma(\alpha+1)^{2}} \\
& -\frac{21600 c^{17} \cosh ^{2}(2 c x) \operatorname{sech}^{6}(c x) \tanh ^{3}(c x) t^{2 \alpha}}{\Gamma(2 \alpha+1)}-\frac{1800 c^{17} \operatorname{sech}^{8}(c x) \sinh (2 c x) t^{2 \alpha}}{\Gamma(2 \alpha+1)} \\
& -\frac{14400 c^{17} \operatorname{sech}^{8}(c x) \tanh (c x) t^{2 \alpha}}{\Gamma(2 \alpha+1)}-\frac{3600 c^{17} \cosh (2 c x) \operatorname{sech}^{6}(c x) \tanh (c x) t^{2 \alpha}}{\Gamma(2 \alpha+1)}
\end{aligned}
$$

$$
\begin{aligned}
& C_{2}=\frac{86400 c^{17} \operatorname{sech}^{6}(c x) \tanh ^{9}(c x) t^{2 \alpha}}{\Gamma(\alpha+1)^{2}}+\frac{5702400 c^{17} \operatorname{sech}^{8}(c x) \tanh ^{7}(c x) t^{2 \alpha}}{\Gamma(\alpha+1)^{2}} \\
& +\frac{26697600 c^{17} \operatorname{sech}^{10}(c x) \tanh ^{5}(c x) t^{2 \alpha}}{\Gamma(\alpha+1)^{2}}+\frac{1728000 c^{17} \operatorname{sech}^{6}(c x) \tanh ^{5}(c x) t^{2 \alpha}}{\Gamma(\alpha+1)^{2}} \\
& +\frac{4320 c^{17} \operatorname{sech}^{4}(c x) \tanh ^{5}(c x) t^{2 \alpha}}{\Gamma(2 \alpha+1)}+\frac{1080 c^{17} \operatorname{sech}^{4}(c x) \sinh (2 c x) \tanh ^{4}(c x) t^{2 \alpha}}{\Gamma(2 \alpha+1)} \\
& +\frac{21081600 c^{17} \operatorname{sech}^{12}(c x) \tanh ^{3}(c x) t^{2 \alpha}}{\Gamma(\alpha+1)^{2}}+\frac{6912000 c^{17} \operatorname{sech}^{8}(c x) \tanh ^{3}(c x) t^{2 \alpha}}{\Gamma(\alpha+1)^{2}} \\
& +\frac{4320 c^{17} \operatorname{sech}^{6}(c x) \tanh ^{3}(c x) t^{2 \alpha}}{\Gamma(2 \alpha+1)}+\frac{2880 c^{17} \cosh (2 c x) \operatorname{sech}^{4}(c x) \tanh ^{3}(c x) t^{2 \alpha}}{\Gamma(2 \alpha+1)} \\
& +\frac{480 c^{17} \operatorname{sech}^{4}(c x) \sinh (2 c x) t^{2 \alpha}}{\Gamma(2 \alpha+1)}+\frac{1440 c^{17} \cosh (2 c x) \operatorname{sech}^{6}(c x) \tanh (c x) t^{2 \alpha}}{\Gamma(2 \alpha+1)} \\
& +\frac{1920 c^{17} \operatorname{sech}^{4}(c x) \tanh (c x) t^{2 \alpha}}{\Gamma(2 \alpha+1)}-\frac{2160 c^{17} \operatorname{sech}^{6}(c x) \tanh ^{11}(c x) t^{2 \alpha}}{\Gamma(\alpha+1)^{2}} \\
& -\frac{267840 c^{17} \operatorname{sech}^{8}(c x) \tanh ^{9}(c x) t^{2 \alpha}}{\Gamma(\alpha+1)^{2}}-\frac{8566560 c^{17} \operatorname{sech}^{10}(c x) \tanh ^{7}(c x) t^{2 \alpha}}{\Gamma(\alpha+1)^{2}} \\
& -\frac{950400 c^{17} \operatorname{sech}^{6}(c x) \tanh ^{7}(c x) t^{2 \alpha}}{\Gamma(\alpha+1)^{2}}-\frac{16338240 c^{17} \operatorname{sech}^{12}(c x) \tanh ^{5}(c x) t^{2 \alpha}}{\Gamma(\alpha+1)^{2}} \\
& -\frac{12268800 c^{17} \operatorname{sech}^{8}(c x) \tanh ^{5}(c x) t^{2 \alpha}}{\Gamma(\alpha+1)^{2}}-\frac{8037360 c^{17} \operatorname{sech}^{14}(c x) \tanh ^{3}(c x) t^{2 \alpha}}{\Gamma(\alpha+1)^{2}} \\
& -\frac{19094400 c^{17} \operatorname{sech}^{10}(c x) \tanh ^{3}(c x) t^{2 \alpha}}{\Gamma(\alpha+1)^{2}}-\frac{864000 c^{17} \operatorname{sech}^{6}(c x) \tanh ^{3}(c x) t^{2 \alpha}}{\Gamma(\alpha+1)^{2}} \\
& -\frac{2160 c^{17} \cosh (2 c x) \operatorname{sech}^{4}(c x) \tanh ^{5}(c x) t^{2 \alpha}}{\Gamma(2 \alpha+1)}-\frac{2160 c^{17} \cosh (2 c x) \operatorname{sech}^{6}(c x) \tanh ^{3}(c x) t^{2 \alpha}}{\Gamma(2 \alpha+1)} \\
& -\frac{5760 c^{17} \operatorname{sech}^{4}(c x) \tanh ^{3}(c x) t^{2 \alpha}}{\Gamma(2 \alpha+1)}-\frac{1440 c^{17} \operatorname{sech}^{4}(c x) \sinh (2 c x) \tanh ^{2}(c x) t^{2 \alpha}}{\Gamma(2 \alpha+1)} \\
& -\frac{2880 c^{17} \operatorname{sech}^{6}(c x) \tanh (c x) t^{2 \alpha}}{\Gamma(2 \alpha+1)}-\frac{960 c^{17} \cosh (2 c x) \operatorname{sech}^{4}(c x) \tanh (c x) t^{2 \alpha}}{\Gamma(2 \alpha+1)},
\end{aligned}
$$




$$
\begin{aligned}
u_{3}= & -\frac{1260 c^{17} \Gamma(2 \alpha+1) t^{3 \alpha} \cosh (4 c x) \tanh (c x) \operatorname{sech}^{8}(c x)}{\Gamma(\alpha+1)^{2} \Gamma(3 \alpha+1)}-\frac{29700 c^{17} \Gamma(2 \alpha+1) t^{3 \alpha} \tanh (c x) \operatorname{sech}^{8}(c x)}{\Gamma(\alpha+1)^{2} \Gamma(3 \alpha+1)} \\
& +\frac{2169 c^{17} t^{3 \alpha} \cosh (4 c x) \tanh (c x) \operatorname{sech}^{8}(c x)}{\Gamma(3 \alpha+1)}+\frac{20880 c^{17} \Gamma(2 \alpha+1) t^{3 \alpha} \cosh (2 c x) \tanh (c x) \operatorname{sech}^{8}(c x)}{\Gamma(\alpha+1)^{2} \Gamma(3 \alpha+1)} \\
& -\frac{73341 c^{17} t^{3 \alpha} \cosh (2 c x) \tanh (c x) \operatorname{sech}^{8}(c x)}{2 \Gamma(3 \alpha+1)}-\frac{3 c^{17} t^{3 \alpha} \cosh (6 c x) \tanh (c x) \operatorname{sech}^{8}(c x)}{2 \Gamma(3 \alpha+1)} \\
& +\frac{51879 c^{17} t^{3 \alpha} \tanh (c x) \operatorname{sech}^{8}(c x)}{\Gamma(3 \alpha+1)} .
\end{aligned}
$$

Thus, the approximate solution of time-fractional Kupershmidt equation (3.7) with the first four terms is:

$$
\begin{aligned}
u(x, t)= & \frac{2080 c^{17} \Gamma(2 \alpha+1) t^{3 \alpha} \cosh (2 c x) \tanh (c x) \operatorname{sech}^{8}(c x)}{\Gamma(\alpha+1)^{2} \Gamma(3 \alpha+1)}-\frac{73341 c^{17} t^{3 \alpha} \cosh (2 c x) \tanh (c x) \operatorname{sech}^{8}(c x)}{2 \Gamma(3 \alpha+1)} \\
& -\frac{29700 c^{17} \Gamma(2 \alpha+1) t^{3 \alpha} \tanh (c x) \operatorname{sech}^{8}(c x)}{\Gamma(\alpha+1)^{2} \Gamma(3 \alpha+1)}-\frac{1260 c^{17} \Gamma(2 \alpha+1) t^{3 \alpha} \cosh (4 c x) \tanh (c x) \operatorname{sech}^{8}(c x)}{\Gamma(\alpha+1)^{2} \Gamma(3 \alpha+1)} \\
& +\frac{2169 c^{17} t^{3 \alpha} \cosh (4 c x) \tanh (c x) \operatorname{sech}^{8}(c x)}{\Gamma(3 \alpha+1)}-\frac{3 c^{17} t^{3 \alpha} \cosh (6 c x) \tanh (c x) \operatorname{sech}^{8}(c x)}{2 \Gamma(3 \alpha+1)} \\
& +\frac{51879 c^{17} t^{3 \alpha} \tanh (c x) \operatorname{sech}^{8}(c x)}{\Gamma(3 \alpha+1)}+\frac{12 c^{12} t^{2 \alpha} \cosh (2 c x) \operatorname{sech}^{4}(c x)}{\Gamma(2 \alpha+1)}-\frac{732 c^{7} t^{\alpha} \tanh (c x) \operatorname{sech}^{6}(c x)}{\Gamma(\alpha+1)} \\
& -\frac{744 c^{7} t^{\alpha} \tanh ^{3}(c x) \operatorname{sech}^{4}(c x)}{\Gamma(\alpha+1)}+\frac{960 c^{7} t^{\alpha} \tanh (c x) \operatorname{sech}^{4}(c x)}{\Gamma(\alpha+1)}-\frac{24 c^{12} t^{2 \alpha} \operatorname{sech}^{4}(c x)}{\Gamma(2 \alpha+1)} \\
& -\frac{12 c^{7} t^{\alpha} \tanh ^{5}(c x) \operatorname{sech}^{2}(c x)}{\Gamma(\alpha+1)}+\frac{240 c^{7} t^{\alpha} \tanh ^{3}(c x) \operatorname{sech}^{2}(c x)}{\Gamma(\alpha+1)} \\
& -\frac{240 c^{7} t^{\alpha} \tanh ^{2}(c x) \operatorname{sech}^{2}(c x)}{\Gamma(\alpha+1)}-6 c^{2} \tanh ^{2}(c x)+4 c^{2} .
\end{aligned}
$$

Set $u(x, t)=u_{\alpha}(x, t)$ and take in particular $c=\frac{1}{2}$, we have:

$$
\begin{aligned}
u_{1}(x, t)= & -\frac{2507 t^{3} \tanh \left(\frac{x}{2}\right) \operatorname{sech}^{8}\left(\frac{x}{2}\right)}{262144}+\frac{3393 t^{3} \cosh (x) \tanh \left(\frac{x}{2}\right) \operatorname{sech}^{8}\left(\frac{x}{2}\right)}{524288}-\frac{t^{3} \cosh (3 x) \tanh \left(\frac{x}{2}\right) \operatorname{sech}^{8}\left(\frac{x}{2}\right)}{524288} \\
& -\frac{117 t^{3} \cosh (2 x) \tanh \left(\frac{x}{2}\right) \operatorname{sech}^{8}\left(\frac{x}{2}\right)}{262144}+\frac{3 t^{2} \cosh (x) \operatorname{sech}^{4}\left(\frac{x}{2}\right)}{2048}-\frac{183}{32} t \tanh \left(\frac{x}{2}\right) \operatorname{sech}^{6}\left(\frac{x}{2}\right) \\
& -\frac{93}{16} t \tanh ^{3}\left(\frac{x}{2}\right) \operatorname{sech}^{4}\left(\frac{x}{2}\right)+\frac{15}{2} t \tanh \left(\frac{x}{2}\right) \operatorname{sech}^{4}\left(\frac{x}{2}\right)-\frac{3}{32} t \tanh ^{5}\left(\frac{x}{2}\right) \operatorname{sech}^{2}\left(\frac{x}{2}\right)-\frac{3 t^{2} \operatorname{sech}^{4}\left(\frac{x}{2}\right)}{1024} \\
& +\frac{15}{8} t \tanh ^{3}\left(\frac{x}{2}\right) \operatorname{sech}^{2}\left(\frac{x}{2}\right)-\frac{15}{8} t \tanh \left(\frac{x}{2}\right) \operatorname{sech}^{2}\left(\frac{x}{2}\right)-\frac{3}{2} \tanh ^{2}\left(\frac{x}{2}\right)+1,
\end{aligned}
$$




$$
\begin{aligned}
u_{\frac{1}{2}}(x, t)= & -\frac{17293 t^{3 / 2} \tanh \left(\frac{x}{2}\right) \operatorname{sech}^{8}\left(\frac{x}{2}\right)}{32768 \sqrt{\pi}}-\frac{2475 t^{3 / 2} \tanh \left(\frac{x}{2}\right) \operatorname{sech}^{8}\left(\frac{x}{2}\right)}{2048 \pi^{3 / 2}}+\frac{435 t^{3 / 2} \cosh (x) \tanh \left(\frac{x}{2}\right) \operatorname{sech}^{8}\left(\frac{x}{2}\right)}{512 \pi^{3 / 2}} \\
& +\frac{723 t^{3 / 2} \cosh (2 x) \tanh \left(\frac{x}{2}\right) \operatorname{sech}^{8}\left(\frac{x}{2}\right)}{32768 \sqrt{\pi}}-\frac{105 t^{3 / 2} \cosh (2 x) \tanh \left(\frac{x}{2}\right) \operatorname{sech}^{8}\left(\frac{x}{2}\right)}{2048 \pi^{3 / 2}}-\frac{3}{512} t \operatorname{sech}^{4}\left(\frac{x}{2}\right) \\
& -\frac{24447 t^{3 / 2} \cosh (x) \tanh \left(\frac{x}{2}\right) \operatorname{sech}^{8}\left(\frac{x}{2}\right)}{65536 \sqrt{\pi}}-\frac{t^{3 / 2} \cosh (3 x) \tanh \left(\frac{x}{2}\right) \operatorname{sech}^{8}\left(\frac{x}{2}\right)}{65536 \sqrt{\pi}}+\frac{3 t \cosh (x) \operatorname{sech}^{4}\left(\frac{x}{2}\right)}{1024} \\
& -\frac{183 \sqrt{t} \tanh \left(\frac{x}{2}\right) \operatorname{sech}^{6}\left(\frac{x}{2}\right)}{16 \sqrt{\pi}}-\frac{93 \sqrt{t} \tanh ^{3}\left(\frac{x}{2}\right) \operatorname{sech}^{4}\left(\frac{x}{2}\right)}{8 \sqrt{\pi}}+\frac{15 \sqrt{t} \tanh \left(\frac{x}{2}\right) \operatorname{sech}^{4}\left(\frac{x}{2}\right)}{\sqrt{\pi}} \\
& -\frac{3 \sqrt{t} \tanh ^{5}\left(\frac{x}{2}\right) \operatorname{sech}^{2}\left(\frac{x}{2}\right)}{16 \sqrt{\pi}}+\frac{15 \sqrt{t} \tanh ^{3}\left(\frac{x}{2}\right) \operatorname{sech}^{2}\left(\frac{x}{2}\right)}{4 \sqrt{\pi}} \\
& -\frac{15 \sqrt{t} \tanh \left(\frac{x}{2}\right) \operatorname{sech}^{2}\left(\frac{x}{2}\right)}{4 \sqrt{\pi}}-\frac{3}{2} \tanh ^{2}\left(\frac{x}{2}\right)+1,
\end{aligned}
$$

$$
\begin{aligned}
u_{\frac{3}{4}}(x, t)= & \frac{t^{3 / 2} \cosh (x) \operatorname{sech}^{4}\left(\frac{x}{2}\right)}{256 \sqrt{\pi}}+\frac{51879 t^{9 / 4} \tanh \left(\frac{x}{2}\right) \operatorname{sech}^{8}\left(\frac{x}{2}\right)}{131072 \Gamma\left(\frac{13}{4}\right)}-\frac{22275 \sqrt{\pi} t^{9 / 4} \tanh \left(\frac{x}{2}\right) \operatorname{sech}^{8}\left(\frac{x}{2}\right)}{131072 \Gamma\left(\frac{7}{4}\right)^{2} \Gamma\left(\frac{13}{4}\right)} \\
& -\frac{183 t^{3 / 4} \tanh \left(\frac{x}{2}\right) \operatorname{sech}^{6}\left(\frac{x}{2}\right)}{32 \Gamma\left(\frac{7}{4}\right)}-\frac{93 t^{3 / 4} \tanh ^{3}\left(\frac{x}{2}\right) \operatorname{sech}^{4}\left(\frac{x}{2}\right)}{16 \Gamma\left(\frac{7}{4}\right)}+\frac{15 t^{3 / 4} \tanh \left(\frac{x}{2}\right) \operatorname{sech}^{4}\left(\frac{x}{2}\right)}{2 \Gamma\left(\frac{7}{4}\right)} \\
& -\frac{3 t^{3 / 4} \tanh ^{5}\left(\frac{x}{2}\right) \operatorname{sech}^{2}\left(\frac{x}{2}\right)}{32 \Gamma\left(\frac{7}{4}\right)}+\frac{15 t^{3 / 4} \tanh ^{3}\left(\frac{x}{2}\right) \operatorname{sech}^{2}\left(\frac{x}{2}\right)}{8 \Gamma\left(\frac{7}{4}\right)}-\frac{15 t^{3 / 4} \tanh \left(\frac{x}{2}\right) \operatorname{sech}^{2}\left(\frac{x}{2}\right)}{8 \Gamma\left(\frac{7}{4}\right)} \\
& +\frac{2169 t^{9 / 4} \cosh (2 x) \tanh \left(\frac{x}{2}\right) \operatorname{sech}^{8}\left(\frac{x}{2}\right)}{131072 \Gamma\left(\frac{13}{4}\right)}+\frac{3915 \sqrt{\pi} t^{9 / 4} \cosh (x) \tanh \left(\frac{x}{2}\right) \operatorname{sech}^{8}\left(\frac{x}{2}\right)}{32768 \Gamma\left(\frac{7}{4}\right)^{2} \Gamma\left(\frac{13}{4}\right)} \\
& -\frac{73341 t^{9 / 4} \cosh (x) \tanh \left(\frac{x}{2}\right) \operatorname{sech}^{8}\left(\frac{x}{2}\right)}{262144 \Gamma\left(\frac{13}{4}\right)}-\frac{3 t^{9 / 4} \cosh (3 x) \tanh \left(\frac{x}{2}\right) \operatorname{sech}^{8}\left(\frac{x}{2}\right)}{262144 \Gamma\left(\frac{13}{4}\right)} \\
& -\frac{945 \sqrt{\pi} t^{9 / 4} \cosh (2 x) \tanh \left(\frac{x}{2}\right) \operatorname{sech}^{8}\left(\frac{x}{2}\right)}{131072 \Gamma\left(\frac{7}{4}\right)^{2} \Gamma\left(\frac{13}{4}\right)}-\frac{t^{3 / 2} \operatorname{sech}^{4}\left(\frac{x}{2}\right)}{128 \sqrt{\pi}}-\frac{3}{2} \tanh ^{2}\left(\frac{x}{2}\right)+1 .
\end{aligned}
$$

Remark 3.1. Under the initial conditions (3.8) with $c=\frac{1}{2}$, if $\alpha=1$ then (3.7) becomes (3.1), and an exact solution is (3.5). 


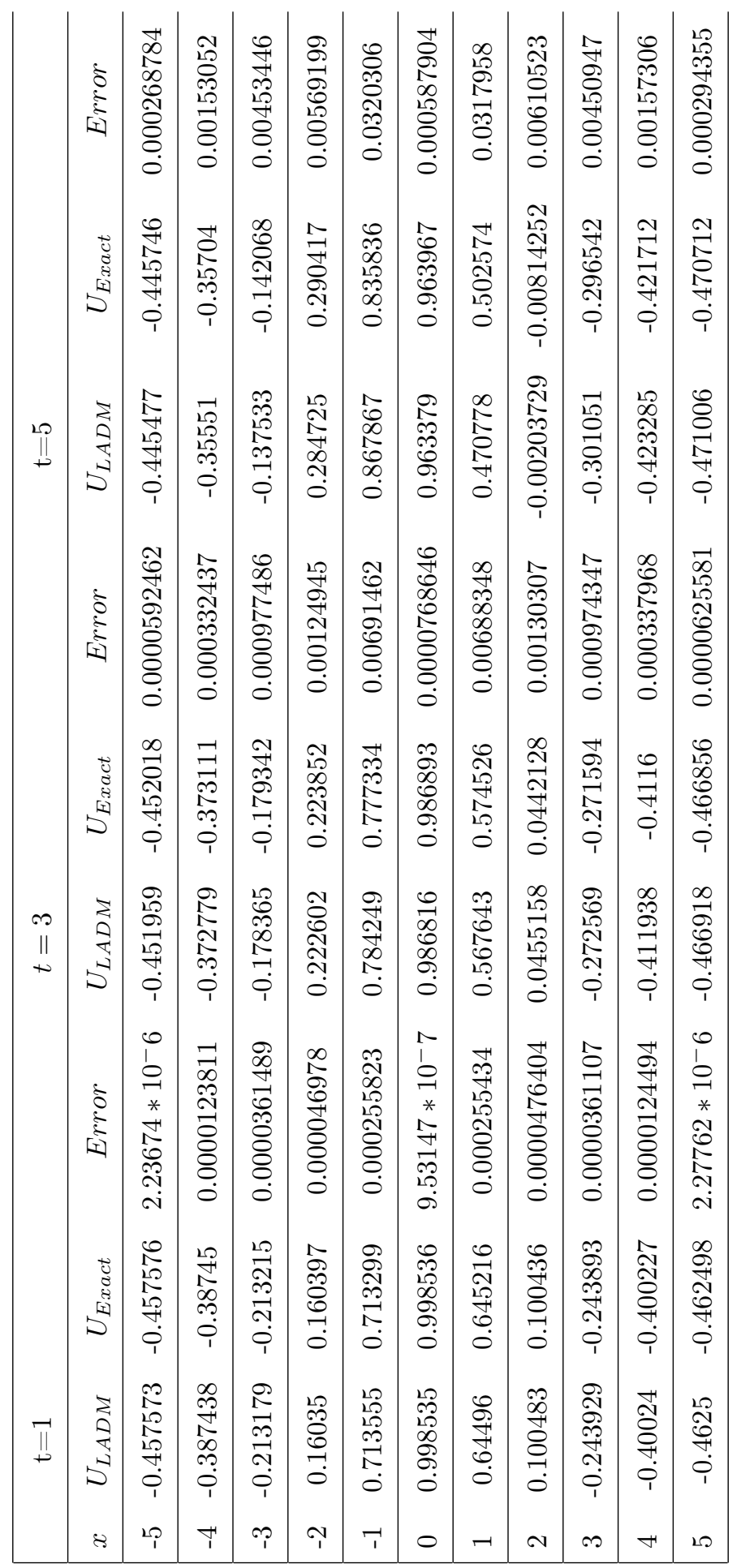

TABLE 1. A comparison between approximate solution and exact solution of (3.1)

$$
\text { for } t=1,3,5 \text {. }
$$




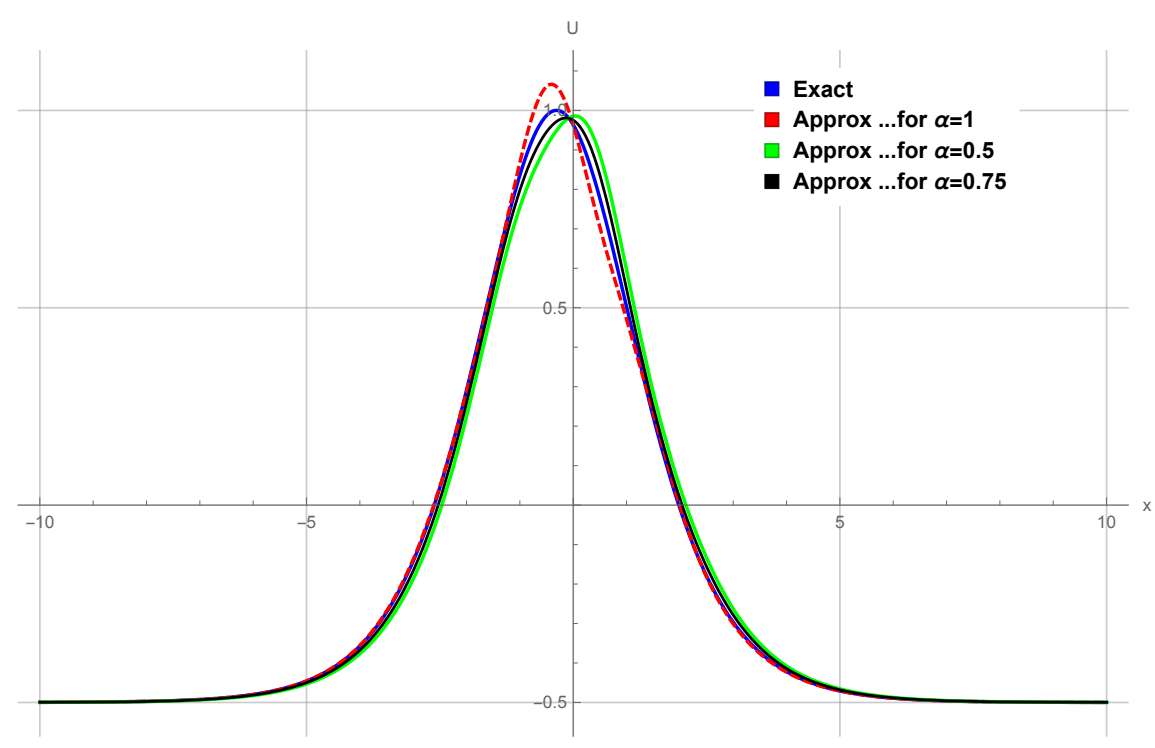

(A) $U_{\text {Exact }}$ and $U_{L A D M}$

Figure 1. Plot of the Exact solution (3.5) of Eq. (3.1) and Approximate solutions of Eq. (3.1), when $c=\frac{1}{2}$ for $t=5$ and $x \in[-10,10]$.

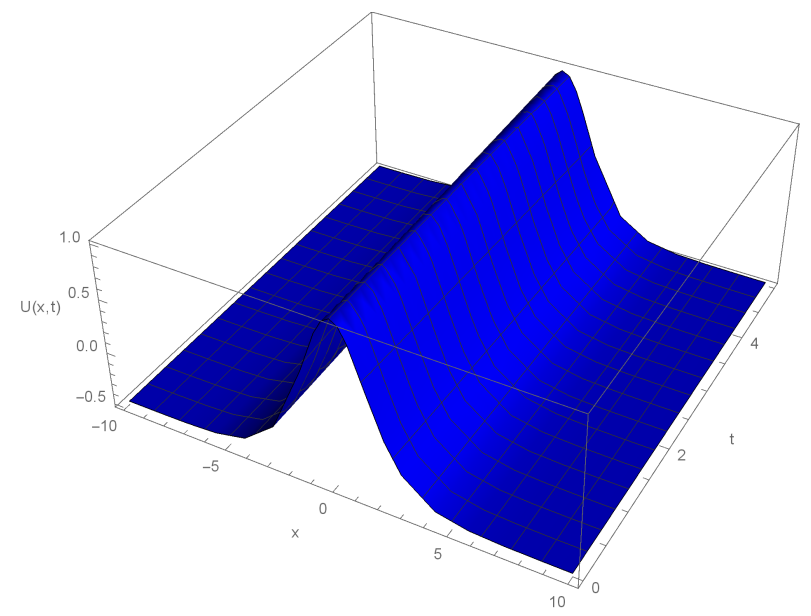

(A) $u(x, t)$

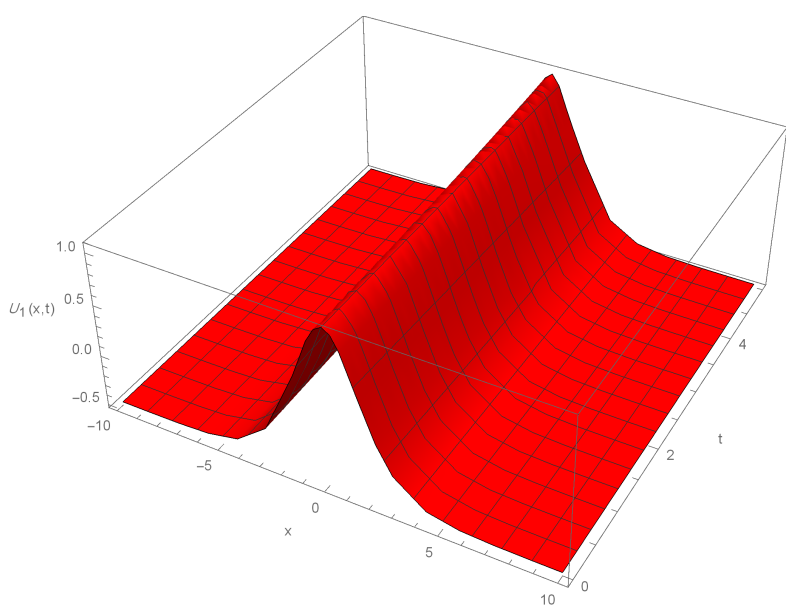

(B) $u_{1}(x, t)$

Figure 2. Plot of the exact solution (3.5) given by Eq. (3.1) and approximate solution $u_{L A D M}$ given by Eq. (3.7) when $\alpha=1$ and $c=\frac{1}{2}$, for $(x, t) \in[-10,10] \times[0,5]$

\section{Conclusion}

In this paper, we discussed three stages related to the study of Kupershmidt equation. First we used the Tanh method to get the exact solution of the equation under study. In the second stage, thanks to the LADM method (ADM in combination with the Laplace transform), we obtain the approximate solutions to the 


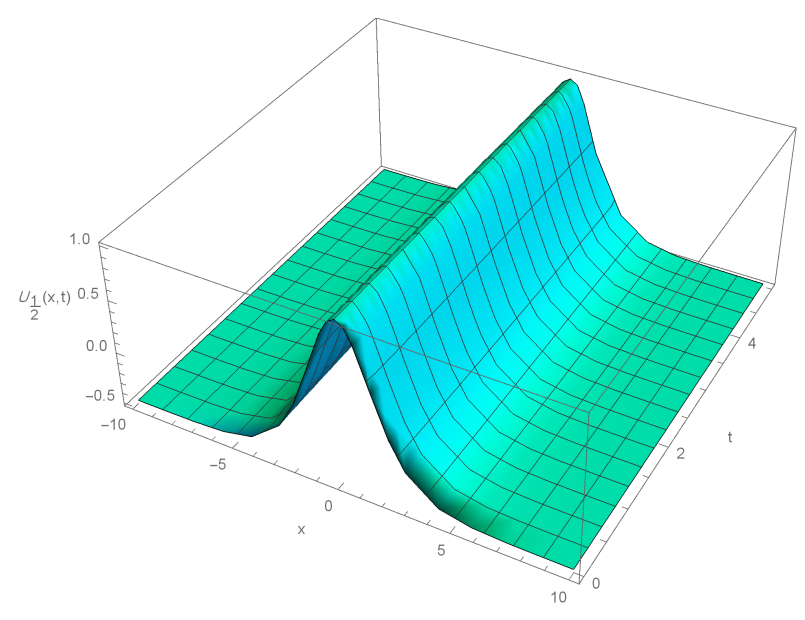

(A) $u_{\frac{1}{2}}(x, t)$



(B) $u_{\frac{3}{4}}(x, t)$

Figure 3. Plot of the approximate solution $u_{L A D M}$

given by Eq. (3.7) when $\alpha=0.5, \alpha=0.75$ and $c=\frac{1}{2}$, for $(x, t) \in[-10,10] \times[0,5]$.

time-fractional Kupershmidt equation. Finally, in order to show the accuracy and efficiency of our method, compare our results with the exact solution of the equation obtained by the Tanh method. Furthermore, we conclude that the LADM is a powerful tool that produces high quality approximate solutions for nonlinear partial differential equations using simple calculations and that attains converge with only few terms.

Conflicts of Interest: The author(s) declare that there are no conflicts of interest regarding the publication of this paper.

\section{REFERENCES}

[1] A. Abdelrazec, D. Pelinovsky, Convergence of the Adomian Decomposition Method for Initial-Value Problems, Numer. Methods Partial Differ. Equations, 27 (2011), 749-766.

[2] G. Adomian, A Review of the Decomposition Method in Applied Mathematics, J. Math. Anal. Appl. 135 (1988), $501-544$.

[3] G. Adomian, System of nonlinear partial differential equations, J. Math. Anal. Appl. 115 (1) (1986), 235-238.

[4] G. Adomian, Solving Frontier Problems of Physics: The Decomposition Method, Kluwer Academic Publication, Boston, 1994.

[5] E. Babolian a, J. Biazar b, A.R. Vahidi, A new computational method for Laplace transforms by decomposition method, Appl. Math. Comput. 150 (2004), 841-846.

[6] E. Babolian, S. Javadi, New method for calculating Adomian polynomials, Appl. Math. Comput. 153 (2004), $253-259$.

[7] K. Charalambous, A. K. Halder and Peter G. L. Leach, A note on analysis of the Kaup-Kupershmidt equation, AIP Conf. Proc. 2153 (2019), 020006.

[8] J. Fadaei, Application of Laplace-Adomian Decomposition Method on Linear and Nonlinear System of PDEs, Appl. Math. Sci. 5 (27) (2011), 1307 - 1315. 
[9] E. Fan, Uniformly constructing a series of explicit exact solutions to nonlinear equations in mathematical physics, Chaos Solitons Fractals, 16 (2003), 819-839.

[10] E. G. Fan, Traveling Wave Solutions for Nonlinear Equations Using Symbolic Computation, Computers Math. Appl. 42 (6-7) (2002), 671-680.

[11] A. Fordy and J. Gibbons, Some Remarkable Nonlinear Transfomations, Phys. Lett. A, 75 (5) (1980), 325.

[12] B. Fuchssteiner, W. Oevel, The bi-Hamiltonlan structure of some nonlinear fifth- and seventh-order differential equatlons and recursion formulas for their symmetries and conserved covarlants, J. Math. Phys. 23 (1982), 358-363.

[13] B. Fuchssteiner, W. Oevel and W. Wiwianka, Computer-Algebra Methods For Investigation Of Hereditary Operators Of Higher Order Soliton Equations, Computer Phys. Commun. 44 (1987), 47-55.

[14] O. G-Gaxiola · J. R. Chávez, R. B-Jaquez, Solution of the Nonlinear Kompaneets Equation Through the Laplace-Adomian Decomposition Method, Int. J. Appl. Comput. Math. 3 (2017), 489-504.

[15] M. A. Helal, M. S. Mehanna, The tanh method and Adomian decomposition method for solving the foam drainage equation, Appl. Math. Comput. 190 (2007), 599-609.

[16] W. Hereman, A. Nuseir, Symbolic methods to construct exact solutions of nonlinear partial differential equations, Math. Computers Simul. 43 (1997), 13-27.

[17] X. B. Hu , D. L. Wang and X. M. Qian , Soliton solutions and symmetries of the $2+1$ dimensional Kaup-Kupershmidt equation, Phys. Lett. A, 262 (1999), 409-415.

[18] M. Hussain and M. Khan, Modified Laplace Decomposition Method, Appl. Math. Sci. 36 (4) (2010), 1769 - 1783.

[19] M. Inc, On numerical soliton solution of the Kaup-Kupershmidt equation and convergence analysis of the decomposition method, Appl. Math. Comput. 172 (2006), 72-85.

[20] K. Jaradat, D. ALoqali, W. Alhabashene, Using Laplace Decomposition Method to Solve Nonlinear Klien-Gordan Equation, U.P.B. Sci. Bull., Ser. D, 80 (2) (2018), 213-222.

[21] K. Khan, M. Ali Akbar, A. H. Arnous, Exact traveling wave solutions for system of nonlinear evolution equations, Springer Plus, 5 (2016), 663.

[22] A. A. Kilbas, H. M. Srivastava, and J. J. Trujillo, Theory and Applications of Fractional Differential Equations, Elsevier, Amsterdam, The Netherlands, 2006.

[23] S. Kumar \& A. Yildirim \& Y. Khanc \& L. Weid, A fractional model of the diffusion equation and its analytical solution using Laplace transform Sci. Iran. 19 (4) (2012), 1117-1123.

[24] W. Malfliet, The tanh method: a tool for solving certain classes of nonlinear evolution and wave equations, J. Comput. Appl. Math. 164-165 (2004), 529-541.

[25] W. Malfliet, The tanh method: A tool for solving certain classes of non-linear PDEs, Math. Meth. Appl. Sci. 28 (2005), 2031-2035.

[26] Z. M. Odibat, S. Momani, Approximate solutions for boundary value problems of time-fractional wave equation, Appl. Math. Comput. 181 (2006), 767-774.

[27] K. B. Oldham and J. Spanier, The Fractional Calculus, Academic Press, New York, NY, USA, 1974.

[28] A. Parker, On soliton solutions of the Kaup-Kupershmidt equation. II. 'Anomalous' N-soliton solutions, Physica D 137 (2000), 34-48.

[29] I. Podlubny, Fractional Differential Equations, Academic Press, New York, NY, USA, 1999.

[30] G. Qingling, Exact Solutions of the mBBM Equation, Appl. Math. Sci. 5 (25) (2011), 1209 - 1215.

[31] S. S. Ray, R. K. Bera, An approximate solution of nonlinear fractional differential equation by Adomians decomposition method, Appl. Math. Comput. 167 (2005), 561-571. 
[32] E. G. Reyes, Nonlocal symmetries and the Kaup-Kupershmidt equation, J. Math. Phys. 46 (2005), 073507.

[33] S. S. Ray, R. K. Bera, Analytical solution of a fractional diffusion equation by Adomian decomposition method, Appl. Math. Comput. 174 (2006), 329-336.

[34] A. H. Salas, Solving the Generalized Kaup-Kupershmidt Equation, Adv. Studi. Theor. Phys. 6 (18) (2012), 879 - 885.

[35] J. L. Schiff, The Laplace Tranform, Theory and Applications, Springer-Verlag, New York, 1999.

[36] M. R. Spiegel, Laplace Tranforms, McGraw-Hill, New York, 1965.

[37] T. Sumbal Shaikh, N. Ahmed, N. Shahid, Z. Iqbal, Solution of the Zabolotskaya-Khokholov Equation by Laplace Decomposition Method, Int. J. Sci. Eng. Res. 9 (2) (2018), 1811-1816.

[38] P. Wang, Bilinear form and soliton solutions for the fifth-order Kaup-Kupershmidt, Mod. Phys. Lett. B, 31 (6) (2017), 1750057.

[39] P. Wang and S. H. Xiao, Soliton solutions for the fifth-order Kaup-Kupershmidt equation, Phys. Scr. 93 (10), 105201.

[40] A. M. Wazwaz, The combined Laplace transform-Adomian decomposition method for handling nonlinear Volterra integrodifferential equations, Appl. Math. Comput. 216 (4) (2010), 1304-1309.

[41] A. M. Wazwaz, A new algorithm for calculating adomian polynomials for nonlinear operators , Appl. Math. Comput. 111 (2000), 53-69.

[42] A. M. Wazwaz, Partial Differential Equations and Solitary Waves Theory, Higher Education Press, Berlin (2009).

[43] L. Yan, Numerical Solutions of Fractional Fokker-Planck Equations Using Iterative Laplace Transform Method, Abstr. Appl. Anal. 2013(2013), Article ID 465160.

[44] Q. Yu , F. Liu, V. Anh and I. Turner, Solving linear and non-linear space-time fractional reaction-diffusion equations by the Adomian decomposition method, Int. J. Numer. Methods Eng. 74 (2008), 138-158.

[45] S. A. Zarea, The tanh method: A tool for solving some mathematical models, Chaos Solitons Fractals, 41 (2009), 979-988. 